\title{
FORECAST HORIZON AND PLANNING HORIZON PATHS IN TIME-INDEXED NETWORK
}

\author{
STANISEAW BYLKA \\ Institute of Computer Science, Polish Academy of Sciences \\ Ordona 21, 01-237 Warszawa, Poland \\ E-mail: bylka@wars.ipipan.waw.pl
}

\begin{abstract}
The problem of existence of a forecast (or planning) horizon has been considered in many special models, more or less precisely. We specify and investigate this problem for families of cheapest paths in networks with weakly ordered nodes. In a discrete network, the standard forward algorithm finds the subnetwork generated by optimal paths. The proposed forward procedure reduces subnetworks such that the forecast horizon remains unchanged. Based on the final subnetwork, we have an answer to the forecast horizon questions. In particular, we show that many questions about rationality of initial decisions become NP-hard. To improve the performance of heuristics, we introduce the notion of potentially rational initial decisions.
\end{abstract}

1. Introduction. The idea of a forecast horizon came from practical management problems when the dynamic parameters are not known for the future. In some cases, a few initial decisions are not affected by future data (beyond a certain period). We consider optimal solutions for a long time horizon with a given forecast window of data. The decisions in the first or first few periods are usually those of immediate importance to managers. Horizon research attempts to quantify the diminishing effect of forecast for future data on initial decisions. The concept of forecast and planning horizons have been formalized in Łoś [1967] for a dynamic programming environment. The paper by Lundin and Morton [1975] initiated research in this direction through the introduction of computational aspect. We refer to Rempała [1991] for the formalization of the horizon concept in the more general settings.

The notion of forecast horizon is closely connected with performance heuristics for nearly optimal solution in a wide variety of dynamic decision problems; see e.g. rolling

2000 Mathematics Subject Classification: 90B50, 90B10, 93C41.

Key words and phrases: cheapest paths problem, forecast and planning horizons, dynamic lot sizing, heuristics.

The paper is in final form and no version of it will be published elsewhere. 
horizon procedure (Lee and Denardo [1986]), receding horizon control (Mayne and Michalska [1990]), repetitive control (Krawczyk and Karacaoglu [1993]) and moving horizon control (Van den Broek [2002]).

The literature on computational results of a forecast horizon procedures is rather limited. Chand and Morton [1986] were the first to develop procedures for determining minimal regeneration sets and minimal forecast horizon (for dynamic lot-size problems). For a given value of $T$, the procedure establishes whether or not $T$ is a forecast horizon. Once the minimal regeneration set has been identified, it only remains to test whether all the adequate optimization problems have a common first period production quantity for at least one optimal solution. In this paper, our exploration goes in the two directions: (1) we concentrate on planning-forecast horizon procedures in more general setting (we don't take into account the nature of the regeneration sets), and (2) we formulate some known and new characterizations for the horizons taking into account the set of decisions reduced by regeneration sets.

Deterministic dynamic optimization problems can be formulated as shortest (cheapest) path problems in a network. Among all classes of problems in network optimization, shortest-path problems have been one of the most extensively studied; see e.g. the monograph of Evans and Minieka [1992], and see also Pochet and Wolsey [1995] for lot sizing problems.

For many problems, depending on available information (a forecast window), there is a "small" set of nodes (a regeneration set) which is visited by optimal paths ended behind the window. At best, such set has only one element, a cut node. In this case, optimal paths end behind the window (longer than a forecast horizon) extending an optimal path to the cut node. As a generalization of these notions, we define a (planning) horizon path with respect to the forecast horizon of a given family of paths in the network. In any case, a forecast window leads to a set of "rational" initial decisions.

The purpose of this paper is to show that the structure of a sub-network generated by the family of optimal paths together with a given regeneration set determinates the existence of a forecast horizon and planning horizon paths.

When we verify the length of a forecast horizon (with respect to our knowledge), we face two problems of a different nature: to find a (minimal) regeneration set and to take potentially rational initial decisions. The first problem is specific for every particular model. The second one can be investigated as a combinatorial problem of a network. Procedures for finding regeneration sets and horizons typically consist of solving problems with increased length until some stopping criterion is satisfied. We refer to Chand and Morton [1986], Bylka, Sethi and Sorger [1992] and Federgruen and Tzur [1995] for a detailed literature review of algorithms in particular dynamic models.

The article is organized as follows. The next section introduces the notation and explains the foundation of the time-indexed networks. In Section 2 we also define the basic notions with respect to subnetworks generated by families of paths. Some properties, which are sufficient for existence of a horizon path with adequate algorithm (also for finding a set of rational initial decisions with respect to the forecast window), are presented in Sections 3 and 4. 


\section{Time-indexed networks (t.i.n.'s)}

2.1. The definition and notation. We use the standard notions of graph theory. $\Re_{+}$ denotes the set of all nonnegative real numbers. The cardinality of a set $S$ will be denoted by $|S|$.

By a network we mean a directed graph with a valuation function - the length - on arcs (sometimes also a cost). In a natural way we extend such functions to paths. For our investigation it is convenient to represent a directed graph by the adjacent list - the multifunction $F$ which lists all predecessors nodes

$$
F: V \rightarrow 2^{V},
$$

where $V$ is the set of nodes. We say that the graph $F$ has $V$ (written also as $V(F)$ ) as the set of nodes and

$$
E(F)=\{(u, v) \mid v \in V(F), u \in F(v)\}
$$

as the set of arcs. Therefore, for each node $u \in F(v)$, the pair $(u, v)$ is an arc of the graph $F$. The multifunction

$$
F^{-1}(v)=\{u \mid v \in F(u)\}
$$

lists all nodes that are attained by an arc from the node $v$.

A graph $F$ is said to be a rooted graph if there exists exactly one node, say $u_{0}$, called the root, such that $F\left(u_{0}\right)=\emptyset$ and $F^{-1}\left(u_{0}\right) \neq \emptyset$. Therefore, we assume:

$$
\text { if } F(u)=\emptyset \text { then } F^{-1}(u)=\emptyset \text { if only } u \neq u_{0} \text {. }
$$

In fact, such a graph consists of a connected graph and a set of isolated nodes.

A function $\tau: V(F) \rightarrow \Re_{+}$such that

$$
\tau(v)-\tau(u) \geq 0 \text { for every }(u, v) \in E(F)
$$

is called a time index of the vertices of the graph $F$. We may think of the length of an arc

$$
l((u, v))=\tau(v)-\tau(u)
$$

as of the "time distance" between its initial and terminal nodes.

Definition 1. We call $(F, \tau)$ the time-indexed network (t.i.n. for short) if

1. $F$ is a rooted graph and $\tau$ a time index of its nodes;

2. For every $x \in \Re_{+}$the set $\{v \in V(F) \mid \tau(v)=x\}$ is finite.

3. There is a lower bound $l^{*}>0$ of the lengths of $\operatorname{arcs}$, i.e. for every $\operatorname{arc}(u, v)$

$$
\text { either } l((u, v)) \geq l^{*} \text { or } l((u, v))=0 \text {. }
$$

$F$ is a locally finite t.i.n. if additionally:

4. $F(v)$ is a finite set for every node $v$.

Time-indexed networks have nodes weakly ordered by "time" (there is a possibility of existence of different nodes with the same time indices). $(F, \tau)$ is a discrete network if $V(F)$ is a discrete set. Without loss of generality, we can assume that $\tau\left(u_{0}\right)=0$ (if not, one can transform the time indices into $\tau(v)-\tau\left(u_{0}\right)$ for every node $v$ ).

By a path in $F$ we mean a sequence of nodes

$$
s=\left(s_{1}, \ldots, s_{n}\right), \quad n \geq 2, \text { such that } s_{i-1} \in F\left(s_{i}\right) \text { for each } i=2, \ldots, n .
$$


The length of $s$ is equal to the sum of the lengths of all its arcs. Therefore $l(s)=$ $\tau\left(s_{n}\right)-\tau\left(s_{1}\right)$ by $(1)$.

For a t.i.n. $F$ we will denote by

$V_{T}(F)$ - the set of nodes $\{v \in V(F) \mid \tau(v)>T$ and $F(v) \neq \emptyset\}$

$P_{u}(F)$ - the set of all paths starting from the node $u$;

$\vec{P}(F)$ - the set of all paths from the root, i.e. $\vec{P}(F)=P_{u_{0}}(F)$;

$P^{W}(F)$ - the set of all paths from the root to a node $v \in W \subset V ; P^{v} \equiv P^{\{v\}}$;

$\vec{P}^{T}(F)$ - the set of all paths from the root and longer than $T$, i.e.

$$
\vec{P}^{T}(F)=P^{V_{T}(F)}(F)=\{s \in \vec{P}(F) \mid l(s)>T\} .
$$

We say that $(A, \tau)$ is a subnetwork of $(F, \tau)$, denoted $A \subset F$, iff

$$
V(A) \subset V(F) \text { and } A(v) \subset F(v) \cap V(A) \text { for each node } v .
$$

We will denote by $F \mid T$ the subnetwork with all arcs which terminate at nodes having the time indices not greater than the number $T$.

We write $A \subset_{T} F$ iff $A \subset F$ and we have the following implication:

$$
\text { if } \tau(v)>T \text { and } P^{v}(F) \neq \emptyset \text { then } P^{v}(A) \neq \emptyset \text {. }
$$

We say that $S \subset F$ is a selection from $F$ iff $F(v) \neq \emptyset$ implies $|S(v)|=1$.

Property 1. Let $F$ be a t.i.n. If $A \subset_{T} F$ is a t.i.n., then every selection $S$ from $A$ is also a t.i.n. and $S \subset_{T} F$.

2.2. Subnetworks generated by families of paths. Families of paths in a t.i.n. can be given arbitrary or constructed by a procedure. For a set $\mathcal{P}$ of paths in a network $F$, the minimal subnetwork $A \subset F$ such that, every path in $\mathcal{P}$ is a path in $A$ (the network generated by $\mathcal{P})$ will be denoted by $\omega_{\mathcal{P}}$. Therefore,

$$
\omega_{\mathcal{P}}(v)=\{u \in F(v) \mid(u, v) \text { forms an arc of a path from } \mathcal{P}\}
$$

and for every $l^{*}<T<T^{\prime}$ we have

$$
\omega_{\vec{P}^{T^{\prime}}(F)} \subset_{T^{\prime}} \omega_{\vec{P}^{T}(F)} \subset_{T} F .
$$

Of course, if $F$ is a connected network then $F=\omega_{\vec{P}(F)}$.

EXAMPLE 1. Now we think of time-indexed networks as networks with two valuation functions (the length and the cost of arcs). We look for structural properties of t.i.n.'s generated by the sets of optimal (cheapest) paths.

Let $(F, \tau)$ be a locally finite t.i.n. and $c: E(F) \rightarrow \Re_{+}$be a cost function on the arcs (and thus on all paths, too). We extend this cost on paths which is the sum of costs of all its arcs.

We deal with problems of finding all $c$-cheapest paths from the root. A simple optimization problem states: Find all paths $s \in P^{v}(F)$ having minimal cost $c(s)$. We will denote by

$\tilde{P}^{v}(F, c)$ - the subset of all cheapest paths in $P^{v}(F)$ and

$\Omega^{c}$ - the subnetwork generated by the family of all $c$-cheapest paths. 


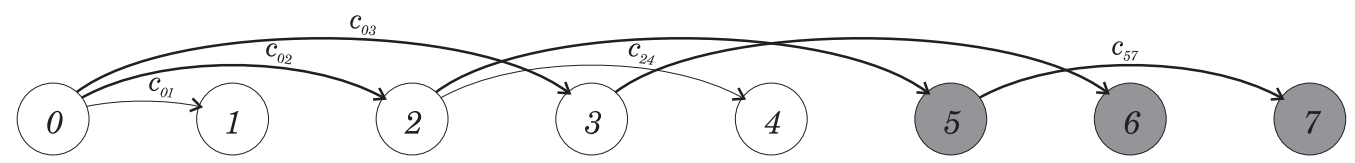

Fig. 1. Exemplifying t.i.n. $\Omega^{c} \mid 7$ with possible 7-regeneration set $R$ (nodes in dark colours) and adequate subnetwork $A \subset_{5} \Omega^{c}$ (bold arcs).

Therefore,

$$
\Omega^{c}=\omega_{\Pi^{c}(F)}, \text { where } \Pi^{c}(F)=\bigcup_{v \in V} \tilde{P}^{v}(F, c)
$$

and for each node $v$ we have:

$$
\Omega^{c}(v)=\left\{u \in F(v) \mid(u, v) \text { forms the last arc of a path } s \in \tilde{P}^{v}(F, c)\right\} .
$$

According to the above notation, consider the following discrete t.i.n.:

$$
F(v)=\{0, \ldots, v-1\} \text { for } v=1,2, \ldots \text { and } F(0)=\emptyset,
$$

with $\tau(v)=v$ and a cost function $c$ given on $F \mid 7$. If, for example, the set of optimal paths $\Pi^{c}(F \mid 7)$ is given by

$$
\begin{array}{c|ccccccc}
v: & 1 & 2 & 3 & 4 & 5 & 6 & 7 \\
\hline P^{v}(F):\{(0,1)\} & \{(0,2)\} & \{(0,3)\} & \{(0,2,4)\} & \{(0,2,5)\} & \{(0,3,6)\} & \{(0,2,5,7)\}
\end{array}
$$

then the induced subnetwork is (see Figure 1) of the form:

$$
\begin{array}{c|cccccccc}
v: & 0 & 1 & 2 & 3 & 4 & 5 & 6 & 7 \\
\hline \Omega^{c}(v): & \emptyset\{0\} & \{0\} & \{0\} & \{2\} & \{2\} & \{3\} & \{5\}
\end{array}
$$

Of course, the subnetwork $\Omega^{c}$ generated by all optimal paths in t.i.n. $(F, \tau, c)$ is also t.i.n. - a subnetwork of $F$. Knowing $c$ only on $\operatorname{arcs}$ of $F \mid T$ we can construct $\Omega^{c} \mid T$ step by step using standard forward procedure.

Let $\gamma_{c}(u, v)$ be the minimal cost of a path, that begins at the root, ends at vertex $v$ with the restriction, that $(u, v)$ is the last arc of the path. Our focus will be the "classical" recurrent equation for the minimal total cost function $\Psi_{c}$ defined on the set of nodes: $\Psi_{c}\left(u_{o}\right)=0$ for the root and for other nodes

$$
\Psi_{c}(v)=\min _{u \in F(v)} \gamma_{c}((u, v))=\min _{u \in F(v)}\left[\Psi_{c}(u)+c((u, v))\right]
$$

and so on

$$
\Omega^{c}(v)=\left\{u \in F(v) \mid \Psi_{c}(v)=\gamma_{c}((u, v))\right\} .
$$

In the case of locally finite network, to find $\Omega^{c} \mid T$, this algorithm requires no more than $O\left(\rho_{T}^{2}\right)$ computations, where $\rho_{T}=|V(F \mid T)|$. See also the discussion in Federgruen and Tzur [1995] for a more general case.

Usually, families of optimal (cheapest) paths are hereditary in the following sense. We say that a set of paths $\mathcal{P}$ is hereditary if both: 
1. Together with a path $\left(s_{1}, \ldots, s_{n}\right)$ it contains $\left(s_{1}, \ldots, s_{i}\right)$ for each $1<i<n$;

2. Together with crossing paths $\left(s_{1}, \ldots, s_{i}, \ldots, s_{i+k}\right)$ and $\left(s_{1}^{\prime}, \ldots, s_{j}^{\prime}, \ldots, s_{j+k^{\prime}}^{\prime}\right)$ such that $s_{i}=s_{j}^{\prime}$ for some $i>1$, it contains each path of the form $\left(s_{1}, \ldots, s_{i}, s_{j+1}^{\prime} \ldots, s_{j+k^{\prime}}^{\prime}\right)$ or $\left(s_{1}^{\prime}, \ldots, s_{j}^{\prime}, s_{i+1} \ldots, s_{i+k}\right)$.

Property 2. If $w \in V(F)$ and $\mathcal{P} \subset P_{w}(F)$ then $\omega_{\mathcal{P}}$ is a t.i.n. with the root $w$. If, additionally, $\mathcal{P}$ is hereditary, then $P_{w}\left(\omega_{\mathcal{P}}\right)=\mathcal{P}$ and

$$
\omega_{\mathcal{P}}(v)=\{u \in F(v) \mid(u, v) \text { forms the last arc of a path from } \mathcal{P}\} .
$$

From now on, we think of a hereditary family of paths as being a time indexed subnetwork of $F$, say $A=\omega_{\mathcal{P}}$ for some $\mathcal{P} \subset \vec{P}(F)$.

2.3. Regeneration sets and planning paths. If a path $s^{\prime}=\left(s_{1}, \ldots, s_{n}, \ldots, s_{n+k}\right), k \geq 0$, then we say that $s^{\prime}$ extends $s=\left(s_{1}, \ldots, s_{n}\right)$ to $s_{n+k}$ or, if only $k>0$, that $\left(s_{n}, \ldots, s_{n+k}\right)$ is a continuation of $s$ to $s_{n+k}$. The set $P_{s_{n}}(F)$ is exactly the set of all continuations of $s$ in $F$.

We say that a path $s=\left(s_{1}, \ldots, s_{n}\right)$ visits a set of nodes $W \subset V(F)$ if it has a node $s_{i}, i>1$, at $W$. We call two paths alternative if both start and terminate at the same nodes.

Definition 2. We say that a set $R \subset V(F \mid T)$ is a $T$-regeneration set of $F$ if every path $s \in \vec{P}^{T}(F)$ (i.e. longer than $T$ ) has an alternative one which visits $R$. It is minimal if $R \backslash\{r\}$ is not a $T$-regeneration set for each $r \in R$.

Let us define another two useful notions: planning set and planning path:

Definition 3. A set of nodes $U \subset F^{-1}\left(u_{0}\right)$ is called a planning set to a set of nodes $W$ in $F$ if for each $v \in W$ there exists a path in $P^{v}(F)$ which visits $U$ and for every $u \in U$ there is a path from $u$ to a vertex in $W$.

A path $s=\left(s_{1}, \ldots, s_{n}\right)$ from the root is said to be a planning path to a set of nodes $W$ in $F$ if there exists $U \subset F^{-1}\left(s_{n}\right)$ which is a planning set to $W$, in the subnetwork $\omega_{P_{s_{n}}(F)}$.

We will denote by

$$
p s_{R}(F)=\left\{U \subset F^{-1}\left(u_{0}\right) \mid U \text { is a minimal planning set to } R \text { in } F\right\} .
$$

It is easy to see the following relationships between planning sets and regeneration sets.

Property 3. Assume that $T$ is large enough, i.e. there is no $v \in V_{T}(F)$ such that $\left(u_{0}, v\right)$ is the only one path to $v$ in F. Then

(i) If $U$ is a planning set to $V_{T}(F)$ then $U$ is a T-regeneration set of $F$ (called also a "planning" T-regeneration set);

(ii) If $R$ is a T-regeneration set of $F$ then every planning set to $R$ in $F$ is also a $T$-regeneration set of $F$;

(iii) If $A \subset_{T} F$ and $R$ is a T-regeneration set of $A$, then $R$ is a $T$-regeneration set of $F$. 
These are some general properties of the notion introduced above.

Property 4. Assume that $A \subset_{H} F$ are t.i.n's, $H \in \Re_{+}$is large enouch and $s^{*}=$ $\left(s_{1}^{*}, \ldots, s_{n}^{*}\right) \in \vec{P}(A \mid H)$ (or $s^{*} \in \vec{P}(F \mid H)$ ). Let us formulate the following statements:

1. a Every path $s \in \vec{P}^{H}(A)$ has an alternative path which extends $s^{*}$ in $A$.

$1 . b$ Every path $s \in \vec{P}^{H}(F)$ has an alternative path which extends $s^{*}$ in $F$.

2. a The path $s^{*}$ is a planning path to the set of nodes $V_{H}(A)$ in $A$.

2.b The path $s^{*}$ is a planning path to the set of nodes $V_{H}(F)$ in $F$.

3. a Each singleton $\left\{s_{i}^{*}\right\}, i=2, \ldots, n$ is an $H$-regeneration set of $A$.

3.b Each singleton $\left\{s_{i}^{*}\right\}, i=2, \ldots, n$ is an $H$-regeneration set of $F$.

4.a The path $s^{*}$ is a planning path to an $H$-regeneration set of $A$.

$4 . b$ The path $s^{*}$ is a planning path to an $H$-regeneration set of $F$.

The statements satisfy:

$4 . a \Rightarrow 1 . a \Leftrightarrow 2 . a \Leftrightarrow 3 a$,

$4 . b \Rightarrow 1 . b \Leftrightarrow 2 . b \Leftrightarrow 3 b$,

and each $k . a \Rightarrow k . b$, for $k=1, \ldots, 4$.

Proof. We have $s^{*} \in P^{\left\{s_{n}\right\}}(A)$. From the definitions, it follows immediately that 1.a $\Rightarrow$ 2. $a \Rightarrow\left\{s_{n}\right\}$ is an $H$-regeneration set of $A \Leftrightarrow 3$. $a$.

On the other hand, if $\left\{s_{n}\right\}$ is an $H$-regeneration set of $A$, then 1.a, because $\vec{P}^{T}(A)$ is hereditary.

Assume 4.a. Let $R$ be an $H$-regeneration set of $A$ such that $s^{*}$ is a planning path to $R$. Let $s^{r} \in P^{r}(A)$ be a path which extends $s^{*}, r \in R$. For every $v \in V_{H}(A)$, there exists a path $p^{v} \in P^{v}(A)$ which visits $R$, say $p^{v}=\left(p_{1}^{v}, \ldots, p_{n_{v}}^{v}\right)$ and $p_{k_{v}}^{v}=r_{v} \in R$, for some $k_{v}<n_{v}$. The path $\left(s^{r_{v}}, p_{k_{v}+1}^{v}, \ldots, p_{n_{v}}^{v}\right) \in P^{v}$ because $P^{v}(A)$ is hereditary. It extends $s^{*}$. This establishes the statement 1.a.

The same proof works for analogous implications for $k . b, k=1, \ldots, 4$.

We have $k . a \Rightarrow k . b$, for $k=1, \ldots, 4$, because $A \subset_{H} F$ and Property 3 .

2.4. Planning and forecast horizons. The notion of forecast horizon was given for families of optimal solutions of dynamic programs in Łoś [1967] and Blikle, Łoś [1967] (and independently, for optimization problems in more specific models, in Lundin and Morton [1975], as well in Bensoussan, Crouhy and Proth [1983]). In this paper we will define the notions of horizons for t.i.n's, only. For an example of the case of finite networks with cycles with positive lengths see Bylka and Sethi [1992].

Our definition is consistent with mentioned above, if optimal solutions in a model are interpreted as cheapest paths.

Definition 4. Let $(F, \tau)$ be a t.i.n. A number $H \in \Re_{+}$is a forecast horizon of a family of paths $\mathcal{P} \subset P_{u}(F), u \in V(F)$, if there exists a path $s^{*} \in P_{u}(F \mid H+\tau(u))$, called an $H$-horizon planning path of $\mathcal{P}$, such that every path $s \in \mathcal{P}, l(s)>H+\tau(u)$, has an alternative path $s^{\prime} \in \mathcal{P}$ which extends $s^{*}$.

The length $l\left(s^{*}\right)=h$ is defined to be a planning horizon of the family $\mathcal{P}$. Then $(h, H)$ is its adequate horizon pair.

If $\mathcal{P}=\vec{P}(F)$, we say that $s^{*}$ is an $H$-horizon planning path of the t.i.n. F. 
Of course, if $s^{*}$ is an $H$-horizon planning path, then $s^{*}$ is an $H^{\prime}$-horizon planning path for every $H^{\prime} \geq H$. If additionally $\mathcal{P}$ is hereditary, $\tilde{s}$ is an $H$-horizon planning path for every $\tilde{s}$ which is alternative to $s^{*}$.

Immediately from the definitions given above we have the following properties of $H$-horizon planning paths:

Property 5. (i) $s^{*}$ is an H-horizon planning path of $F$ if and only if $s^{*}$ is a planning path to the set of nodes $V_{H}(F)$ in $F$ (i.e. 2.b of Property 4).

(ii) If $s^{*}$ is an $H$-horizon planning path of $\mathcal{P}$, then $s^{*}$ is an $H$-horizon planning path of $\omega_{\mathcal{P}}$. The reverse implication is also true for hereditary $\mathcal{P}$.

If, additionally, $u$ is the root of $F$ and $\omega_{\mathcal{P}} \subset_{H} F$, then $s^{*}$ is an H-horizon planning path of $F$.

Łoś defines the planning path as a single arc path. The Lundin and Morton definition uses arbitrary paths as above. If $\mathcal{P}$ is a set of paths, which extend $s^{*}$, then $l\left(s^{*}\right)=h=H$ is a forecast as well as a planning horizon (see Bensoussan, Crouhy and Proth [1983] for this special case) of $\mathcal{P}$.

2.5. Horizons of subnetworks generated by regeneration sets. A subnetwork $F$ reduced by a regeneration set $R$, denoted by $R F$, is defined as the subnetwork generated by the set of all paths from the root going through $R$ and longer than $T$, i.e.:

$$
R F=\omega_{\mathcal{P}} \text { where } \mathcal{P}=\left\{s \in \vec{P}^{T}(F) \mid s \text { visits } R\right\} .
$$

Property 6. Let $R$ be a T-regeneration set of a t.i.n. F. We have

$$
R F \subset_{T} F
$$

and

$$
p s_{R}(F)=p s_{R}(F \mid T)=p s_{R}(R F \mid T) .
$$

Additionally, each element of $\operatorname{ps}_{R}(F)$ is a planning T-regeneration set of $F$.

For a $T$-regeneration set of $F$, by Property 4 , if a singleton $\{q\} \in p s_{R} F$, then the one arc path $s^{*}=\left(u_{0}, q\right)$ is a $T$-horizon planning path of $F$. For more general case we have the following theorem.

Theorem 1. Let $A \subset_{H} F$ and $s^{*}$ be a path from the root in $A \mid H$, say $s^{*} \in P^{\{q\}}(A)$. The following statements are equivalent:

(i) $s^{*}$ is an $H$-horizon planning path of $A$;

(ii) The singleton $\{q\}$ forms an $H$-regeneration set of $A$;

(iii) There exists a selection $S$ from $A$ such that $s^{*}$ is an $H$-horizon planning path of $S$.

Each of $(i)-($ iii $)$ is a consequence of:

(iv) $R$ is an $H$-regeneration set of $A$ and $s^{*}$ is a planning path to $R$.

Additionally, each of $(i)-($ iii) implies

$(v) s^{*}$ is an H-horizon planning path of $F$. 
Proof. We see at once that $(i) \Leftrightarrow(i i) \Rightarrow(v)$ and $(i v) \Rightarrow(i)$, which is clear from Property 4 (because $(i) \Leftrightarrow 1 . a$ and $(i v) \Leftrightarrow 4 . a)$.

Assume (i). We construct the selection $S$ which satisfies (iii). Consider the set of paths

$$
\mathcal{P}=\left\{s \in \vec{P}^{H}(A) \mid s \text { extends } s^{*}\right\} .
$$

Let $S$ be a selection from the network $\omega_{\mathcal{P}} . S$ is a selection from $A$ and $s^{*}$ is an $H$-horizon planning path of the family of paths $\vec{P}(S)$. Hence, $S=\omega_{\vec{P}(S)}$ and, by Property $5($ ii), we have $($ iii).

Assume (iii). The statement (i) follows from Property 4 because $s^{*}$ is an $H$-horizon planning path of $S \subset_{H} A$.

From (iii) of Theorem 1, we conclude that:

COROLLARY 1. The problem of the existence of a planning-forecast horizon pair (or an $H$-horizon planning path) of a time indexed network is NP hard, even of the t.i.n. is locally finite and discrete.

We investigate the class of optimization problems with limited information about the future. To verify if our knowledge leads to a "good" initial decision, we ask about existence of a planning-forecast horizon pair. In particular, one looks for the minimal forecast horizon of families of solutions of optimization problems in a considered model (see Chand and Morton [1986], Federgruen, Tzur [1995] and Bylka, Sethi [1992]).

In a particular model we ask about a collection of assumptions which guarantee the existence of optimal plans as well as regeneration sets. In the presented general setting, we simply assume the existence of optimal plans - cheapest paths. Additionally, we assume that the set of all optimal paths is hereditary.

3. Algorithm for horizon planning paths. In this section it is required that considered networks are locally finite.

3.1. Some specific regeneration sets. The notion of $T$-regeneration set of a t.i.n. was given in Definition 2 with a specification in Properties 3 and 6 given as a planning $T$-regeneration set. In fact, we are interested in other more specific $T$-regeneration sets. We call a $T$-regeneration set $R$ to be $T$-cut if every path longer than $T$ visits $R$.

Let us denote:

$$
C u t_{T} F=F\left(V_{T}(F)\right) \backslash V_{T}(F)=\left\{u \in V(F \mid T) \mid u \in F(v) \text { for some } v \in V_{T}(F)\right\} .
$$

It is the "over- $T$ " minimal cut of $F$. If the root $u_{0} \notin C u t_{T} F$ then it is a $T$-regeneration set of $F$.

Definition 5. A $T$-regeneration set $R$ of $F$ is called an over-T regeneration set if $R \subset$ $\mathrm{Cut}_{T} F$. It is called adequate if, additionally,

$$
F^{-1}(R) \cap V_{T}(F)=F^{-1}\left(C u t_{T} R F\right) \cap V_{T}(F) .
$$

Of course, every $T$-regeneration set $R$ of $F$ is a cut of the t.i.n. $R F$ (i.e. of the subnetwork $F$ reduced by $R$ ). On the other hand, the set $C u t_{T} R F$ is an over- $T$ regeneration set of $F$. 
Generally, we have

$$
R \subset C u t_{T} R F \subset C u t_{T} F \text { for any over-T regeneration set } R \text { of } F .
$$

For suitable networks we have

$$
R F \subset_{T}\left(C u t_{T} R F\right) F \text { for any } T \text {-regeneration set } R \text { of } F \text {. }
$$

In particular, if $R$ is a $T$-cut set of $F$ then

$$
R F(v)=F(v) \text { for any } v \in V_{T}(F) .
$$

Property 7. Let $R$ be an over-T cut set of a t.i.n. F. Then

(i) $R$ is a T-regeneration set of $A$ for any t.i.n. $A \subset_{T} F$;

(ii) For every $T^{\prime}>T$ and $T^{\prime}$-regeneration set $R^{\prime} \subset R \cup V_{T}(F)$ we have

$$
R^{\prime} F=R^{\prime} R F \text {. }
$$

Additionally, for every selection $S$ from $R F$

$$
\tilde{F}(v)= \begin{cases}S(v) & \text { if } \tau(v) \leq T \\ F(v) \cap\left(V_{T}(F) \cup R\right) & \text { otherwise }\end{cases}
$$

is a t.i.n. and

$$
R^{\prime} \text { is a } T^{\prime} \text {-regeneration set of } F \Leftrightarrow R^{\prime} \text { is a } T^{\prime} \text {-regeneration set of } \tilde{F} \text {. }
$$

It is desired, in specific models, that a sequence of $T$-cut sets should be done (on the basis of our knowledge about the future). In this aspect, Property 7(ii) can be utilized step by step.

For relations between forecast horizons and regeneration sets, we have the following useful corollary of Theorem 1 and Property 7 .

COROLlary 2. Let $R$ be an $H$-regeneration set of a t.i.n. F. The following three statements are equivalent:

(i) $H$ is a forecast horizon of $R F$;

(ii) There exist an over-H regeneration set $\tilde{R}$ of $R F$ and a set $U \in p_{\tilde{R}}(R F)$ such that $|U|=1$

(iii) There exists a selection $S$ from $R F$ such that $H$ is a forecast horizon of $S$.

Additionally, if $R$ is an $H$-cut set of $F$, then $(i)$ takes a more general form:

$\left(i^{\prime}\right) H$ is a forecast horizon of any t.i.n. $A \subset_{H} F$.

REMARK 1. In order to verify whether $T$ is a forecast horizon of a t.i.n. $F$, we can verify it on a subnetwork $R F$ through its selections. If $R$ is a $T$-cut of $F$, then this reduction is perfect, i.e. the answer with respect to existence is the same for $F$ and for $R F$.

3.2. The cancelling operators. Let $F$ be a t.i.n. and $R$ be a set of nodes of $F \mid T$. We will consider the behaviour of the "cancelling" operator $\Phi_{T, R}$, which applied to $F$ yields a subnetwork of $F$. We define them in the following way.

Denote

$$
Z_{T, R}(F)=\{v \in V(F \mid T) \mid v \notin R\} .
$$


First, we define

$$
\phi_{T, R} F(v)= \begin{cases}\emptyset & \text { if }(F \mid T)^{-1}(v)=\emptyset \text { and } v \in Z_{T, R}(F), \\ F(v) \backslash Z_{T, R}(F) & \text { if } \tau(v)>T \\ F(v) & \text { otherwise. }\end{cases}
$$

The operator $\phi_{T, R}$ eliminates all arcs of $F \mid T$ which terminate at "dead ends" (i.e. at nodes from which no arc starts and which do not belong to $R$ ). The $m$-th iteration of $\phi_{T, R}$ will be denoted by $\phi_{T, R}^{m}$. It is clear that

$$
\phi_{T, R}^{m+1} F \subset_{T} \phi_{T, R}^{m} F .
$$

Finally, we obtain the operator

$$
\Phi_{T, R} F=\lim _{m \rightarrow \infty} \phi_{T, R}^{m} F .
$$

In the locally finite t.i.n. case we have the equality for some $\bar{m}$, i.e.

$$
\Phi_{T, R} F=\phi_{T, R}^{\bar{m}+1} F=\phi_{T, R}^{\bar{m}} F .
$$

Property 8. Let $R$ be a T-regeneration set of a t.i.n. F. Then $\Phi_{T, R}(F)(v) \neq \emptyset$ if and only if either there exists a path from $v$ to $R$ in $F$ or there exists $s=\left(s_{1}, \ldots, s_{n}\right) \in S^{v}(F)$ such that $s_{i} \in R$ and $\tau\left(s_{i+1}\right)>T$ for some $i \leq n$. We have

$$
\Phi_{T, R}(F)(v)=F(v) \backslash Z_{T, R}(F) \text { if only } \Phi_{T, R}(v) \neq \emptyset \text {. }
$$

\subsection{The main properties of the cancelling concepts}

LEMmA 1. If $R$ is a T-regeneration set of a t.i.n. $F$, then $\Phi_{T, R}(F \mid T)$ is a t.i.n. Additionally, if $R$ is an adequate over- $T$ regeneration set, then $\Phi_{T, R} F$ is a t.i.n. such that $\Phi_{T, R}(F \mid T)=\left(\Phi_{T, R} F\right) \mid T$.

Proof. Assume $\Phi_{T, R} F^{-1}(v) \neq \emptyset$ for a vertex $v \in V(R F)$.

If $\tau(v) \leq T$, then $\Phi_{T, R} F(v)=F(v) \neq \emptyset$ by the definition of the operator $\Phi_{T, R}$. Therefore $\Phi_{T, R}(F \mid T)$ is a t.i.n.

Suppose contrary to our claim that $\tau(v)>T$ and $\Phi_{T, R} F(v)=\emptyset$. Then $F(v) \subset Z_{R}$ and so on $v \notin F^{-1}(R)$ in spite of (3). Therefore, $\Phi_{T, R} F$ is a t.i.n., also.

Assume $\tau(v) \leq T$. If there exists a path from $v$ to $R$ in $F$ then $\Phi_{T, R}(F)(v)=$ $F(v)=\Phi_{T, R}(F \mid T)(v)$, by Property 8. In the opposite case $\Phi_{T, R}(F)(v)=\emptyset$ as well as $\Phi_{T, R}(F \mid T)(v)=\emptyset$.

THEOREM 2. If $R$ is an adequate over-T regeneration set, then $\Phi_{T, R} F$ is a t.i.n. such that

$$
\Phi_{T, R} F \subset_{T} R F \text { with equality if, additionally, } R=\text { Cut }_{T} R F .
$$

Proof. By Lemma $1, \Phi_{T, R} F$ is a t.i.n.

If $u \in \Phi_{T, R}(F)(v)$ then $u \in R F(v)$, by Property 8 .

Assume $u \in R F(v)$. It follows that $(u, v)$ forms the last arc in a path which visits $R$. From (3) and Property 8, it follows that $\Phi_{T, R}(F)(v) \neq \emptyset$. If $\tau(u)>T$ then $u \in$ $\Phi_{T, R}(F)(v)$, because $u \notin Z_{T, R}(F)$ and by Property 8 .

If $\tau(u) \leq T$ and $R=C \operatorname{tat}_{T}(R F)$ then $u \in \Phi_{T, R}(F)(v)$. In the opposite case, by (3), there exists $r \in R$ such that $v \in F^{-1}(r)$. Therefore, $\Phi_{T, R}(F)(v) \neq \emptyset$ and the proof is complete. 
THEOREM 3. Let $R$ be a T-regeneration set of a t.i.n. F. We have

$$
p s_{R} F \subset\left\{B^{-1}\left(u_{0}\right) \mid B \in \varphi_{R} F\right\},
$$

where

$$
\varphi_{R} F=\left\{\Phi_{T, R} S \mid S \text { is a selection from } \Phi_{T, R}(F \mid T)\right\} .
$$

Proof. Assume $U \in p s_{R}(F)$. Thus, $U \subset F^{-1}\left(u_{0}\right)$ and $U$ is a minimal planning set to $R$. Let us define the following sets of paths:

$$
\mathcal{P}=\left\{s \in \vec{P}^{T}(R F) \mid s \text { visits } U\right\} .
$$

and denote $A=\omega_{\mathcal{P}}$. We have $A \subset_{T} R F$, because $U$ is a $T$-regeneration set of $R F$. This clearly forces

$$
A \subset_{T} F \text { and } \Phi_{T, R} A\left|T \subset \Phi_{T, R} F\right| T .
$$

From the construction of $A$ we obtain $(A \mid T)^{-1}\left(u_{0}\right)=U$. From minimality of $U$ we have

$$
\left(\Phi_{T, R} \tilde{W}\right)^{-1}\left(u_{0}\right)=U
$$

for every selection $\tilde{W}$ from $A \mid T$.

Let $\tilde{W}$ be a selection from $\Phi_{T, R} A \mid T$. It can be improved to a selection $S$ from $\Phi_{T, R} F \mid T$ such that $\tilde{S}(v)=S(v)$ if only $\tilde{S}(v) \neq \emptyset$. We have $\Phi_{T, R} S=\Phi_{T, R} \tilde{S}$. From (6), we have the desired inclusion.

3.4. The algorithm. Here and subsequently the symbol $T|F| T^{\prime}$, for $T^{\prime}>T$, denotes the multifunction $F$ restricted to the set of nodes $V\left(T|F| T^{\prime}\right)=\left\{v \in V(F) \mid T<\tau(v) \leq T^{\prime}\right\}$. The following properties (consequences of Property 7 and Corollary 2) of the cancelling operators are useful from computational point of view.

Property 9. Let $R$ be a $T$-cut set of $F$ and $R^{\prime} \subset R \cup V\left(T|F| T^{\prime}\right)$ be a $T^{\prime}$-regeneration set of $F$, where $T^{\prime}>T$. Then

(i) $\Phi_{T^{\prime}, R^{\prime}} \Phi_{T, R} F=\Phi_{T^{\prime}, R^{\prime}} F$.

(ii) If $U \in p s_{R}(F)$, then there exists $U^{\prime} \subset U$ such that $U^{\prime} \in p s_{R^{\prime}}\left(\Phi_{T, R} F\right) \subset p s_{R^{\prime}}(F)$.

(iii) If $\tilde{S} \in \varphi_{R}(F), S^{\prime}$ is a selection from $T|F| T^{\prime}$ and

$$
S(v)= \begin{cases}\tilde{S}(v) & \text { if } \tau(v) \leq T, \\ S^{\prime}(v) & \text { otherwise, }\end{cases}
$$

then $S$ is a t.i.n. and $\Phi_{R^{\prime}} S \in \varphi_{R^{\prime}}(F)$.

In fact instead of the assumption " $R$ be a $T$-cut set of $F$ ", we can take $R$ being an adequate over- $T$ regeneration set of $F$ (for some restrictions see Remark 1 ).

The properties of the cancelling operators suggest the following procedure for choosing a potentially rational initial arc (possibly planning horizon arc).

\section{Procedure Select}

STEP 0. We start with a subnetwork $F \mid T$ having the root $u_{0}$ and with a $T$-cut (or an over- $T$ regeneration set) $R$.

STEP 1. Use the cancelling operator to find the subnetwork $\Phi_{T, R}(F \mid T)$. If the set $\left[\Phi_{T, R}(F \mid T)\right]^{-1}\left(u_{0}\right)$ is a singleton, say $\left\{u_{1}\right\}$, take $s^{*}=\left(u_{0}, u_{1}\right)$ and go to End. Otherwise, go to Step 2. 
STEP 2. Look for a selection $S$ from $\Phi_{T, R}(F \mid T)$ such that $\left(\Phi_{T, R} S\right)^{-1}\left(u_{0}\right)$ is a singleton. We may exploit Property 9 (iii) in the case given $t$-regeneration set $R_{t}$ and $\varphi_{R_{t}}(F)$ for some $t<T$. If such a selection, say $S^{*}$, is found, then take $s^{*}=\left(u_{0}, u_{1}, \ldots, u_{k}\right)$ such that

$$
\left[\Phi_{T, R}\left(S^{*}\right)\right]^{-1}\left(u_{i-1}\right)=\left\{u_{i}\right\} \text { for } i=1, \ldots, k \text { and }\left|\left[\Phi_{T, R}\left(S^{*}\right)\right]^{-1}\left(u_{k}\right)\right|>1,
$$

and go to End. In the other case, complete $\varphi_{R}(F \mid T)$ and go to End.

END. We constructed $\Phi_{R, T}(F \mid T)$ and either $s^{*}=\left(u_{0}, u_{1}, \ldots, u_{k}\right), k \geq 1$ given explicitly together with an adequate selection $S^{*}$ or $\varphi_{R}(F)$. In the last case we can construct $p s_{R}(F)$ (the family of planning sets to $R$ ) and $S^{*} \in \varphi_{R}(F)$.

Consider the case where the output of the procedure is a family $\varphi_{R}(F)$ without singletons in $p s_{R}(F)$. We can choose $S^{*} \in \varphi_{R}(F)$ such that

$$
\left|S^{*-1}\left(s_{0}\right)\right|=\min _{U \in p s_{R}(F)}|U|,
$$

or by any other procedure. If the first decision has to be chosen, define $s^{*}=\left(u_{0}, u_{1}\right)$ with $u_{1} \in S^{*-1}\left(s_{0}\right)$. It will be potentially rational.

If we obtain a new information as a $T^{\prime}$-regeneration set, then we ought to ask about an initial decision in an updated network. Suppose that in a consecutive period $T^{\prime}>T$ we obtain an extension of the subnetwork as $T|F| T^{\prime}$ and an over- $T^{\prime}$-regeneration set $R^{\prime} \subset R \cup V\left(T|F| T^{\prime}\right.$ ) (with respect to new information). We consider two possibilities: either an initial decision was taken or it wasn't. In each case we use Procedure Update and replay Procedure Select with updated subnetwork $F^{\prime} \mid T^{\prime}$ and $R^{\prime}$.

\section{Procedure Update}

STEP 0. We start with an output of Procedure Select; a subnetwork $\Phi_{T, R} F \mid T$ and an extension of the subnetwork $T|F| T^{\prime}$ for some $T^{\prime}>T$. If an initial decision was taken, go to Step 1. Otherwise, go to End.

STEP 1. We have $S^{*}$ and $s^{*}=\left(u_{0}, u_{1}, \ldots, u_{k}\right)$ with $k \geq 1$. We improve $S^{*}$ by taking

$$
\tilde{S}(v)= \begin{cases}\left\{u_{1}\right\} & \text { if } v \in S^{*-1}\left(S^{*-1}\left(u_{0}\right)\right), \\ S(v) & \text { otherwise. }\end{cases}
$$

It changes $S^{*}$ in the case $k=1$ and $S^{*-1}\left(u_{0}\right)$ has more than one element.

The node $u_{k}$ becomes the root of $F^{\prime}$, such that $F^{\prime}\left(u_{k}\right)=\emptyset$ and for other nodes

$$
F^{\prime}(v)= \begin{cases}\tilde{S}(v) & \text { if } \tau\left(u_{k}\right) \leq \tau(v) \leq T, \\ F(v) \backslash Z_{T, R} & \text { otherwise. }\end{cases}
$$

Define

$$
u_{0}^{\prime}=u_{k} \text { and } \tau^{\prime}(v)=\tau(v)-\tau\left(u_{k}\right) \text { for } v \in V\left(F^{\prime}\right)
$$

and go to End.

END. We have $F^{\prime} \mid T^{\prime}$ obtained in Step 1. Otherwise, define $F^{\prime}\left|T^{\prime}=F\right| T^{\prime}$.

The algorithm is a generalization of the solution procedures given by Chand and Morton [1986] for the Wagner-Whitin model and Bylka, Sethi and Sorger [1992] for an equipment replacement model. It yields a horizon pair if there exists a forecast horizon. 
In each case, the algorithm constructs a set of potentially rational initial decisions with respect to the forecast window.

4. Examples. The example given below illustrates a possibility how optimization problems in a dynamic model can be transformed into equivalent cheapest path problems in a t.i.n.

EXAMPLE 2. Consider the following inventory model with continuous demand and discrete replenishment. The demand appears with a given rate $D(t)$ at continuous time $t$, and all demand must be met. Every sequence $\left(t_{0}, \ldots, t_{n}\right)$ where $0=t_{0} \leq t_{1} \leq \ldots \leq t_{n}=T$ represents a replenishment schedule on $[0, T]$ such that in each $t_{i-1}, i=1, \ldots, n$, the size of the replenishment is equal to the cumulative demands in the time interval $\left[t_{i-1}, t_{i}\right]$, i.e.

$$
W_{t_{i-1}}^{t_{i}}=\int_{t_{i-1}}^{t_{i}} D(t) d t .
$$

For a given fixed cost of replenishment $K>0$ and stock holding cost per item per time $h$, the cost of the schedule is equal to

$$
c\left(t_{0}, \ldots, t_{n}\right)=n K+h \sum_{i=1}^{n} Z_{t_{i-1}}^{t_{i}},
$$

where

$$
Z_{u}^{v}=\int_{u}^{v}\left(W_{u}^{v}-W_{u}^{t}\right) d t
$$

is the cumulative holding inventory in the time interval $[u, v]$. For any given $T$, we want to determine a replenishment schedule with the minimal cost. It is easy to see that if $T$ is not too small than we have the condition 3 of Definition 1, i.e. there is a $l^{*}$ such that $t_{i}-t_{i-1} \geq l^{*}$

Consider a t.i.n. $F$ with $\Re_{+}$as the set of nodes and

$$
E=\left\{(u, v) \in \Re_{+} \times \Re_{+} \mid v-u \geq l^{*} \text { and ether } u=0 \text { or } u \geq l^{*}\right\}
$$

as the set of arcs. We have $F(v)=\left\{u \in \Re_{+} \mid u \leq v-l^{*}\right.$ either $u=0$ or $\left.u \geq l^{*}\right\}$, with time indices $\tau(v)=v$ and arc costs $c(u, v)=K+h Z_{u}^{v}$.

Every path $\left(t_{0}, \ldots, t_{n}\right)$ from the root $t_{0}=0$ to $t_{n}=T$ represents a replenishment schedule having the cost

$$
c\left(t_{0}, \ldots, t_{n}\right)=\sum_{i=1}^{n} c\left(t_{i-1}, t_{i}\right) .
$$

For a given $T$ we look for all cheapest path of the length $T$ (optimal paths from the root 0 to the node $T$ ) in $F$.

The case of piecewise constant demand rate $D$ is presented in Bylka and Rempała [2004].

According to (more general) Example 1, we can investigate families of optimization problems. The limited information means that instead of $c$ from $\mathcal{C}$ we know $c \mid T$ i.e. the costs of all arcs in the subnetwork $F \mid T$. Such a cost is called a T-forecast cost. The 
additional information is contained in the set of all possible extensions of $c \mid T$ to cost functions from $\mathcal{C}$, i.e.:

$$
c|T| \mathcal{C}=\{d \in \mathcal{C} \mid d((u, v))=c((u, v)) \text { if only } \tau(v) \leq T\} .
$$

For families of optimization problems, given by $c \in \mathcal{C}$ in $F$, we ask if $c \mid T$ constitutes a sufficient knowledge to find a forecast horizon and horizon planning path (see Definition 4). The same sytuation we have for every family of optimal paths $\Pi^{d}(F)$ for $d \in c|T| \mathcal{C}$.

Definition 6. Consider a family of optimization problems $c|H| \mathcal{C}$ in $F$.

(i) We say that a path $s^{*}$ is an $H$-horizon planning path for the family $c|H| \mathcal{C}$ if it is an $H$-horizon planning path of $\Omega^{d}$ for every $d \in c|H| \mathcal{C}$.

(ii) An arc from the root is said to be a potentially rational initial decision if it is the first arc of a $d$-cheapest longer than $H$ path for a cost $d \in c|H| \mathcal{C}$.

A set $U_{0} \subset F^{-1}\left(u_{0}\right)$ is said to be a sufficient planning set for $c|H| \mathcal{C}$, if it is a planning set to $V_{H}\left(\Omega^{d}\right)$ in any $\Omega^{d}$, if only $d \in c|H| \mathcal{C}$. Additionally, $U_{0}$ ought to be minimal.

The second concept given above is a "rationality" of initial decision with respect to our knowledge. Of course, we shall be interested to find such sets $U_{0}$ as small as possible. In particular, if $U_{0}=\{q\}$ then the one arc path $s^{*}=\left(u_{0}, q\right)$ is an $H$-horizon planning path of the family of optimization problems.

The information about the network $F$ and the cost function given by $c|T| \mathcal{C}$ with respect to the future, can be transformed to $T$-regeneration sets. The main question is, to find a specifical $T$-regeneration set $R$ of the t.i.n. $\Omega^{c|T| \mathcal{C}}$, where

$$
\Omega^{c|T| \mathcal{C}}(v)=\bigcup_{d \in c|T| \mathcal{C}} \Omega^{d}(v) .
$$

Namely, $R$ ought to be a $T$-regeneration set of

$$
\Omega^{d} \subset_{T} \Omega^{c|T| \mathcal{C}} \text { for every } d \in c|H| \mathcal{C} .
$$

A cut of $\Omega^{c|T| \mathcal{C}}$ can be used as such common regeneration set.

EXAMPLE 2 (continued). We are concerned with a constant demand rate

$$
D(t)=a \text { for some } a>0, K>0 \text { and } h=1 .
$$

There is the case where $\mathcal{C}=\{c\}$. The forecast window as well as the family of optimization problems are determined by its length only.

We have: $Z_{0}^{u}=\frac{1}{2} a u^{2}$ and for costs

$$
c(v, v+t)=c(0, t)=K+\frac{1}{2} a t^{2} .
$$

There is exactly one arc, say $\left(0, t^{*}\right)$, from the root with minimal average cost. Furthermore, $t^{*}=\sqrt{\frac{2 K}{a}}$, with average cost $\sqrt{2 K a}$ and $c\left(0, t^{*}\right)=2 K$.

All replenishment problems of the length $T=n t^{*}$ or $T=\infty$ have average cost $\sqrt{2 K a}$ and optimal paths contain only arcs of length $t^{*}$. Let us denote such a path with $n$ arcs by $\left(0, t^{*}\right)^{n}$. It is easy to check that, in general, in optimal paths all arcs have the same length. Additionally, for every $T=n t^{*}+\alpha$, where $n>0$ and $0 \leq \alpha<t^{*}$, there 
is exactly one optimal path. From Theorem 1 in Bylka and Rempała [2004] we have $\tilde{P}^{\{T\}}(F, c)=\{s(T)\}$, with

$$
s(T)= \begin{cases}\left(0, t^{*}+\frac{\alpha}{n}\right)^{n} & \text { if } \alpha \leq r_{n} t^{*}, \\ \left(0, t^{*}-\frac{t^{*}-\alpha}{n+1}\right)^{n+1} & \text { if } \alpha \leq r_{n} t^{*},\end{cases}
$$

where $r_{n}=\sqrt{n^{2}+n}-n$. In this case $\Omega^{c}=\Omega^{c|T| \mathcal{C}}$ and $\left|\Omega^{c}(v)\right|=1$ for every node $v \in \Re_{+}$. For every $T=n t^{*}+\alpha$, as above, the interval

$$
R_{T}=\left(T-t^{*}-\max \left\{\frac{\alpha}{n}, \frac{t^{*}-\alpha}{n+1}\right\}, T\right]
$$

is a $T$-regeneration set of $\Omega^{c}$. There is exactly one selection from $\Omega^{c}$. Therefore, with respect to Theorem 1, there is no forecast horizon planning path for this family of problems. The set

$$
U_{0}=\left(t^{*} \sqrt{1-\frac{1}{n+1}}, t^{*} \sqrt{1+\frac{1}{n}}\right)
$$

is a planning set to $V_{T}\left(\Omega^{c}\right)$ sufficient for potentially rational initial decisions.

REMARK 2. In discrete networks, we have common $T$-regeneration sets for $\Omega^{d}, d \in c|T| \mathcal{C}$, in each of the following two cases:

- Every arc of $\Omega^{c|T| \mathcal{C}}$ has the length not greater than a number, say $k^{*}$, and $T>k^{*}$. Then the set

$$
R_{T}=\left\{v \in V\left(\Omega^{c|T| \mathcal{C}}\right) \mid T-k^{*}<\tau(v) \leq T\right\}
$$

is a cut of $\Omega^{c|T| \mathcal{C}}$ (as in Example 2);

- There are no two $\operatorname{arcs}\left(u^{\prime}, v^{\prime}\right)$ and $\left(u^{\prime \prime}, v^{\prime \prime}\right)$ in $\Omega^{c|T| \mathcal{C}}$ such that $\tau\left(u^{\prime \prime}\right)<\tau\left(u^{\prime}\right)<$ $\tau\left(v^{\prime}\right)<\tau\left(v^{\prime \prime}\right)$ and $u \in \Omega^{c|T| \mathcal{C}}(v)$ with $\tau(v)=T$ then the set

$$
R_{T}=\left\{w \in V\left(\Omega^{c|T| \mathcal{C}}\right) \mid \tau(u) \leq \tau(w) \leq T\right\}
$$

is a cut of $\Omega^{c|T| \mathcal{C}}$.

The last case is typical for models of Wagner and Whitin type. Generally, in models which admitted capacity for productions or stocks, the subnetworks generated by families of optimal paths do not satisfy conditions given above.

A sequence of $T$-regeneration sets for $T=t_{0}, t_{0}+1, \ldots$, say $\left(R_{t_{0}}, R_{t_{0}+1}, \ldots\right)$ is called monotonic iff for every $t \geq t_{0}$ we have

$$
R_{t+1} \subset R_{t} \cup\{v \mid \tau(v)=t+1\} .
$$

In models without capacities, monotonic sequences of minimal cut sets $R_{t}$ for $t=t_{0}, t_{0}+$ $1, \ldots$ (with $t_{0}$ as small as possible) are algorithmically constructed in Chand and Morton [1986] and Bylka, Sethi and Sorger [1992]. The perfect lot-size procedure given by Bastian [1992] constructs the sequence of minimal cut-regeneration sets to obtain the minimal forecast horizon. In every case, such sequence makes a possibility to define a perfect procedure for finding planning and forecast horizons.

According to Definition 6(ii), the relation between sets of potentially rational initial decisions and regeneration sets is established by the following theorem. 
THEOREM 4. Consider a family of optimization problems $c|H| \mathcal{C}$ in $F$. Let $R$ be an adequate over $H$-regeneration set of $\Omega^{c|H| \mathcal{C}}$.

(i) There exists a selection $S$ from $\Omega^{c} \mid H$ such that $\left(\Phi_{H, R} S\right)^{-1}\left(u_{0}\right)$ is a sufficient planning set for $(c|H| \mathcal{C})$.

(ii) If there exists a selection $S$ from $\Omega^{c} \mid H$ such that any path to $R$ in $\Phi_{H, R} S$ is an extension of $s^{*} \in \vec{P}\left(\Omega^{c} \mid H\right)$, then $s^{*}$ is an $H$-horizon planning path for the family $c|H| \mathcal{C}$. If $R$ is a cut set of $\Omega^{c|H| \mathcal{C}}$, then we have the opposite implication also.

Proof. Property 7(i) now shows that $R$ is an adequate over $H$-regeneration set of $\Omega^{d}$ for each $d \in c|H| \mathcal{C}$. The first statement of the theorem is a consequence of Theorem 3 , because $\Omega^{d}\left|H=\Omega^{c}\right| H$.

The second statement of the theorem is a simplification of the implication $(i v) \Rightarrow(i)$ in Theorem 1 , because the same $\{q\}$ is an $H$-regeneration set of each $\Omega^{d}$.

Let $s^{*} \in S^{q}\left(\Omega^{c} \mid H\right)$. If $R$ is a cut set of $\Omega^{c|H| \mathcal{C}}$, then $s^{*} \in \vec{P}\left(R \Omega^{c|H| \mathcal{C}}\right)$ and we may find appropriate $S$.

EXAMPLE 3. Consider the problem of determination of the schedule that minimizes the total operating cost in a discrete equipment replacement model with multiple technologies. A replacement policy determines when to sale an old machine and change it to another one from a set of technological possibilities $M=\{1, \ldots, \bar{m}\}$.

We look at t.i.n. $F$ with the set of nodes $V=M \times \mathcal{N}$, time index $\tau(m, t)=t$ and

$$
F(m, t)= \begin{cases}\left\{\left(m, t^{\prime}\right) \mid t^{\prime}<t\right\} \cup\left\{\left(m^{\prime}, t\right) \mid m^{\prime} \neq m\right\} & \text { if } m>0, \\ \left\{\left(m^{\prime}, t\right) \mid m^{\prime} \neq m\right\} & \text { if } m=0 .\end{cases}
$$

- An arc of the form $e=\left(\left(m, t^{\prime}\right),(m, t)\right)$ represents the decision to buy machine of technology $m$ at the beginning of period $t^{\prime}+1$, to utilize it and to sale at the end of period $t$. The cost $c(e)$ consists of the purchase cost minus the salvage value resale machine and the sum of operation costs in periods $t^{\prime}+1, \ldots, t$.

- An arc of the form $\left(\left(m^{\prime}, t\right),(m, t)\right), m>0$, is related to switch between different technologies with adequate cost at period $t$.

- The node $u_{0}=(0,0)$ is the root of the t.i.n. $F$. An arc of the form $((0,0),(m, 0))$ is related to the beginning of the program-decision with respect to technology of the machine buying at the beginning of the first period. Arc of the form $((m, t),(0, t))$ for $t>0$, is related to the end of the program (we have $F^{-1}(0, t)=\emptyset$ ). Each such arc $e$ has the cost $c(e)=0$.

The production plant under consideration starts its business in period 1 and goes out of business at the end of period $T$. Each path from the root $(0,0)$ to the node $(0, T)$ defines a possible replacement plan. The problem of minimizing this cost over all admissible plans is equivalent to the problem of cheapest patch. Bylka, Sethi and Sorger [1992] develop methods that can be used to check whether a forecast horizon exists in a certain family of "monotonic" costs $\mathcal{M}$. Their numerical example leads to the t.i.n. $\Omega^{c} \mid 8$, presented in Fig. 2, with over 8-regeneration set $R_{8}$ (nodes in dark colours) adequate to the family of subnetworks $\Omega^{d}, d \in c|8| \mathcal{M}$. 


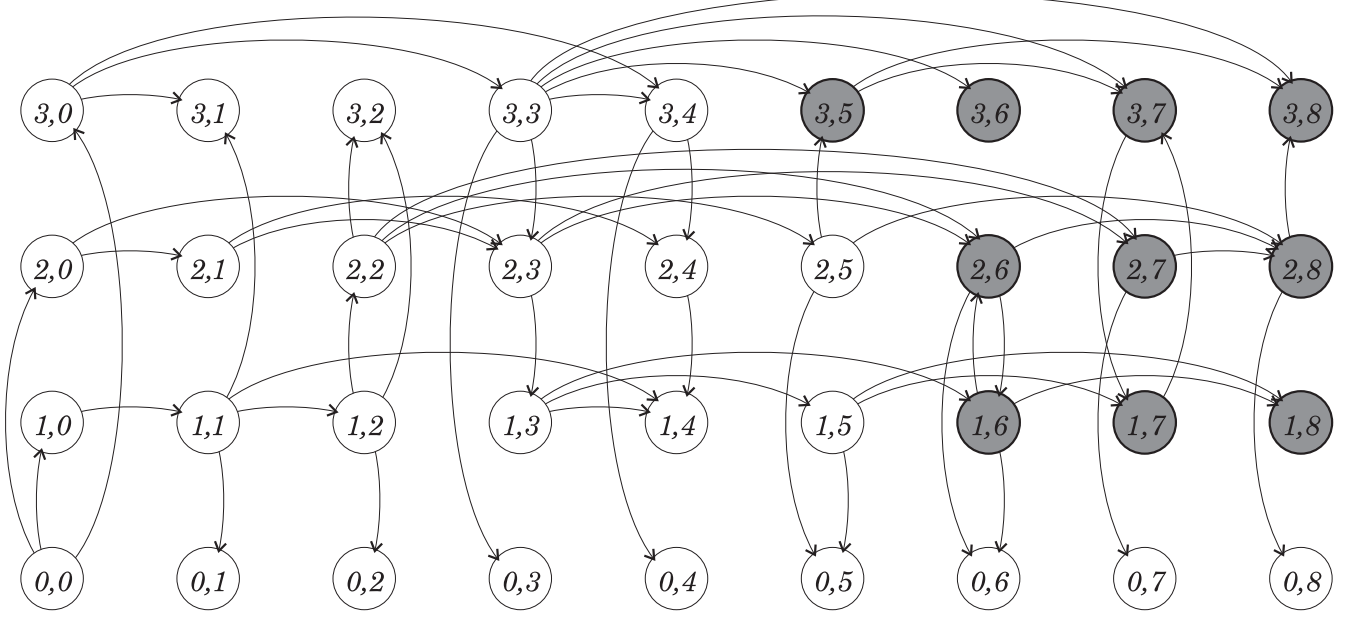

Fig. 2. The t.i.n. $\Omega^{c} \mid 8$ with over8-regeneration set $R_{8}$ (nodes in dark colours) adequate for the family of subnetworks in Example 3.

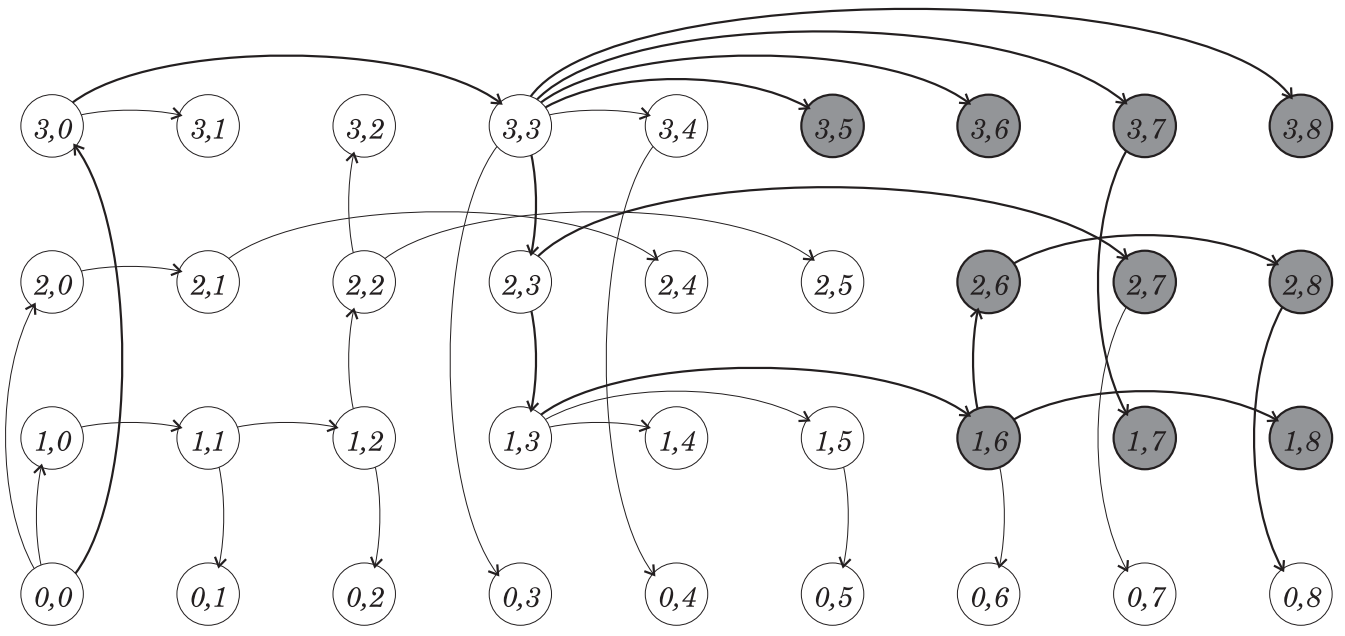

Fig. 3. The subnetworks $S$ and $\Phi_{8, R_{8}} S$ (illustrated by bold arcs)

We use the Algorithm to verify the existence of horizon planning paths searched selections from the t.i.n. $\Omega^{c} \mid$ 8. Look at $S$ presented in Fig. 3 . It is easy to check that each path in $\Phi_{8, R_{8}} S$ (illustrated by bold arcs) is an extension of the path $s^{*}=((0,0),(3,0),(3,3))$. Therefore, by Theorem 3 (ii), $H=8$ is a forecast horizon with $s^{*}$ as a horizon planning path.

The algorithm is a generalization of the solution procedures given by Chand and Morton [1986] for the Wagner-Whitin model and Bylka, Sethi and Sorger [1992] for an equipment replacement model. It obtains a horizon pair if there exists a forecast horizon. In each case, the algorithm constructs a set of potentially rational initial decisions with respect to the forecast window. 
5. Concluding remarks. The results can be used to improve known heuristics in selecting lot size quantities. Each heuristic has its own formula for choosing a decision-an arc from $A^{-1}\left(u_{0}\right)$. The general idea is to start with a chosen heuristic and improve it in the following way (see Bylka [1999]):

- For a given forecast window, say up to the period $T$, find a $T$-cut set $R$ and construct the sufficient planning set $U$, of potentially rational initial decisions with respect to $R$ (see Definition 6 and Theorem 4).

- Use a given heuristic for choosing a decision from $U$.

It is still an open problem when the improved heuristics have a narrower error band. The algorithm looks for a minimal forecast horizon. Procedures to detect a maximal planning horizon for a given forecast window in a family of inventory control problems have been recently developed by Bensoussan et al. [1983]. The presented procedure can be easily improved to obtain a maximal horizon planning path.

\section{References}

M. Bastian [1992], A perfect lot-tree procedure for discounted dynamic lot-size problem with speculation, Naval Research Logistics 39, 651-668.

A. Bensoussan, M. Crouhy and J. M. Proth [1983], Mathematical Theory of Production Planning, Advanced Series in Management, Vol. 3, North-Holland.

A. Blikle and J. Łoś [1967], Horizons in dynamic programs with continuous time, Bulletin de L'Académie Polonaise des Sciences, Math., Astron. Phys. 15, 513-519.

S. Bylka and S. Sethi [1992], Existence and derivation of forecast horizon in a dynamic lot-size model with nondecreasing holding costs, Production and Operations Management 1, 212-224.

S. Bylka, S. Sethi and G. Sorger [1992], Minimal forecast horizons in equipment replacement models with multiple technologies and general switching costs. Naval Research Logistics 39, 487-507.

S. Bylka [1999], Improving rolling horizon heuristics for dynamic lot-sizing problems, in: Inventory Modeling - A selection of research papers presented at the fourth ISIR Summer School, R. Hill and D. Smith (eds.), University of Exeter, 13-19.

S. Bylka and R. Rempała [2004], Heuristics for impulse replanishment with continuous periodic demand, International Journal of Production Economics 88, 183-190.

S. Chand and T. E. Morton [1986], Minimal forecast horizon procedures for dynamic lot-size model, Naval Research Logistics Quarterly 33, 111-122.

J. R. Evans and E. Minieka [1992], Optimization Algorithms for Networks and Graphs, Dekker, 1992.

A. A. Federgruen and M. Tzur [1995], Fast solution of minimal forecast horizons in dynamic programs with single indicator of the future: applications to dynamic lot-sizing models, Management Science $41874-893$.

J. B. Krawczyk and G. Karacaoglu [1993], On repetitive control and the behaviour of a middleaged consumer, European Journal of Operational Research 66, 89-99.

C. Y. Lee and E. Denardo [1986], Rolling planning horizon: Error bounds for the dynamic lot size model, Mathematics Operations Research 11, 423-432. 
J. Łoś [1967], Horizons in dynamic programs, in: Proc. of the Fifth Berkeley Symp. On Mathematical Statistics and Probability, California University Press, Berkeley, CA, 479-490.

R. Lundin and T. Morton [1975], Planning horizon for the dynamic lot size model: zabel vs. protective procedures and computational results, Operations Research 23, 711-735.

D. Q. Mayne and H. Michalska [1990], Receding horizon control of nonlinear systems, IEEE Transactions on Automatic Control 35, 814-824.

Y. Pochet and L. A. Wolsey [1995], Algorithms and reformulations for lot sizing problems, DIMACS Series in Discrete Mathematics and Theoretical Computer Science 20, 245-293.

R. Rempała [1991], Forecast horizon in a dynamic family of one-dimensional control problems, Dissertationes Mathematicae 315.

W. Van den Broek [2002], Moving horizon control in dynamic games, Journal of Economic Dynamic and Control 26, 937-961. 